\title{
CROUZON SYNDROME: A CASE REPORT AND REVIEW OF LITERATURE
}

\author{
Harroop Kaur,* Harmeet Singh Waraich,** Chander Mohan Sharma, ***
}

\begin{abstract}
Crouzon syndrome, also called craniofacial dysostosis is an autosomal dominant disorder characterized by premature closure of cranial sutures, midfacial hypoplasia and orbital defects. Herein we report a case of this rare entity who presented with brachycephaly, maxillary hypoplasia, wide parrot beaked nose, repaired bilateral cleft lip and cleft palate along with dental and orbital abnormalities.
\end{abstract}

Keywords: Crouzon syndrome, craniosynostosis, maxillary hypoplasia.

\section{INTRODUCTION}

Crouzon syndrome is a rare genetic disorder with autosomal dominant inheritance with the prevalence of 1 in 25,000 live births, and it constitutes $4.8 \%$ of all craniosynostosis. In $25 \%$ of cases, it may also occur sporadically because of a fresh mutation. The underlying pathological process is premature synostosis of the coronal, sagittal and occasionally lambdoid sutures beginning in the first year of life and completed by 2-3 years of life. This fusion does not allow the bones to grow normally, affecting the shape of the head, appearance of the face and the relationship of the teeth.The diagnosis is based on clinical findings and radiological examination. This syndrome was described by Crouzon in 1912 who described a patient with a characteristic group of deformities which were then observed in other individuals. Crouzon syndrome is caused by malformations of the mesenchyme and ectoderm. Mutation of the gene for fibroblast growth factor receptor 2 (FGFR2) is responsible for Crouzon syndrome and this gene has been mapped to the long arm of chromosome 10 and mutations in exon $B$ of FGFR2 gene have been described ${ }^{[1]}$.

\section{CASE REPORT}

A 14 year old male patient presented with non specific complaints of post nasal catarrh and headache for the last 10 days. After detailed clinical and radiological examination, he was diagnosed as a case of Crouzon syndrome with negative family history.

On local examination, the patient had a brachycephalic head formation, maxillary hypoplasia, a wide parrot beaked nose with deviated nasal septum and rhinolalia. There was mild conductive hearing loss $(30 \mathrm{~dB})$ in the left ear. The patient had bilateral cleft lip and cleft palate for which he was operated at the age of 3 years. On dental examination, he had abnormal dental occlusion and maxillomandibular relation. On ophthalmic examination, the patient had bilateral proptosis, strabismus with esotropia, and high myopia. He had hypertelorism and there was evidence of healed exposure keratopathy in the left eye with bilateral Bitot spots. Fundus examination revealed myopic degeneration in both the eyes.
The patient had a dark complexion. Anthropometry was normal.Cardiovascular system, central nervous system, respiratory and abdominal examination was normal. Routine hematological and biochemical tests were within normal limits.

\section{DISCUSSION}

The main clinical feature of Crouzon syndrome is a skull deformity which may be brachycephaly, oxycephaly or trigonocephaly. Hydrocephalus and mental retardation may be present because of premature fusion of cranial sutures ${ }^{[2]}$. There is maxillary hypoplasia with relative mandibular prognathism and dental malocclusion. There can be deviation of the nasal septum, narrowed or obliterated anterior nares, wide parrot beaked nose and rhinolalia. Midfacial anomalies like cleft lip and cleft palate might be present. Hearing loss may be there which is usually conductive. Orbital defects include bilateral proptosis because of shallowing of orbits secondary to arrested growth of the maxilla and zygoma, and anterior positioning of the greater wing of the sphenoid.

There might be spontaneous luxation of the globes also. There is strabismus (esotropia or exotropia) and hypertelorism. There might be vision threatening complications like exposure keratopathy and optic atrophy due to compression of the optic foramen. Acanthosis nigricans can also be associated with Crouzon syndrome. Patients of Crouzon syndrome are usually dark complexioned.

In radiological examination, anteroposterior, lateral and cephalometric views of the skull are taken. It shows premature suture closure and provides information about maxillomandibular relation. 'Paw marking' of the skull is seen due to raised intracranial pressure ${ }^{[3]}$. CT scan confirms the standard radiographical findings and provides information on ventricular size. Suture closure can be graphically displayed by 3-dimensional CT scans. Cephalometric studies measure the dimensions of some functional spaces like orbits, rhinopharynx and nasal cavities ${ }^{[4]}$.

Management of a patient of Crouzon syndrome has two components:

*Junior Resident, ***Professor and Head, Department of Otolaryngology and Head and Neck Surgery,**Junior Resident, Dept of Opthomology I.G.Medical College, Shimla. 


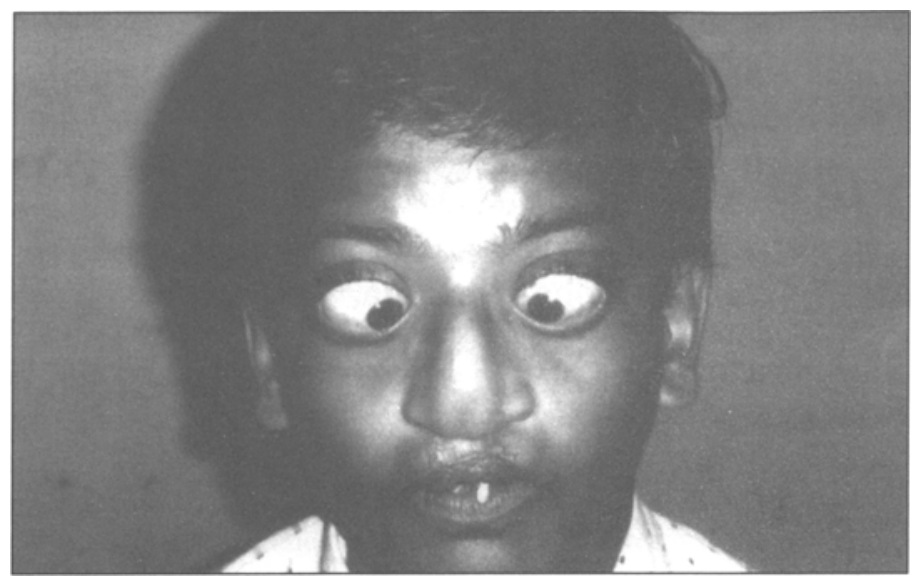

Fig 1: Photograph of the patient showing repaired bilateral cleft lip,malocclusion of teeth,wide parrot beaked nose,bilateral proptosis and esotropia.

1) First is the release of prematurely fused sutures based on evidence of raised intracranial pressure. Surgery is mainly carried out early after 3-6 months [5]. The principle is the release of bony ankylosis by exposure of fused sutures via a coronal flap.

2) Craniofacial reconstructive surgery including advancement of the maxilla and frontonasal complex; and other surgeries depending upon the deformities in the patient like rhinoplasty, oculoplasty and cleft lip and cleft palate repair can be done.

Early and accurate diagnosis of a patient of Crouzon syndrome is essential. Genetic counselling plays an important role. The need, extent and timing of treatment depend upon the severity of the disease and age of the patient. For complete evaluation, optimum treatment planning and comprehensive services, a multidisciplinary approach to the management of a patient of Crouzon syndrome is needed.

\section{REFERENCES}

1. Malcolm S,Reardon W.Fibroblast growth factor receptor-2 mutations in craniosynostosis. Ann NY Acad Sci 1996;785:164-70.

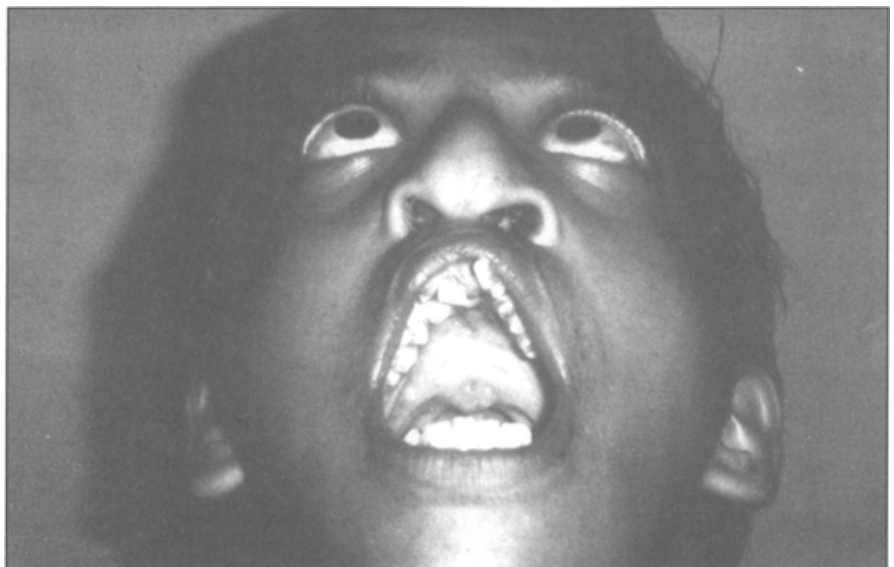

Fig 2 : Photograph of the patient showing repaired cleft palate, dental malposition and maxillary hypplasia.

2. Noetzel MJ, Marsh JL, Palkes H,Gado M.Hydrocephalus \& mental retardation in craniosynostosis. J Pediatr 1985;107(6):885-92.

3. David Lisa R, Velotta Emily, Weaver R Grey, Wilson John A, Argenta Louis C.Clinical findings precede objective diagnostic testing in the identification of increased intracranial pressure in syndromic craniosynostosis. Journal of Craniofacial Surgery 2002;13(5):67680 .

4. Carinci F, Avantaggiato A,Curioni C.Crouzon syndrome:Cephalometric analysis and evaluation of pathogenesis.Cleft Palate Craniofac J 1994;31(3):201-9.

5. Di Rocco C, Marchese E, Velardi F.Craniosynostosis:surgical treatment during the first year of life. J Neurosurg Sci 1992; 36(3): 129-37.

Address for correspondence :

Prof. Chander Mohan Sharma, B-39, Sector 2, Lane I, New Shimla-171009, Shimla.(H.P)

\section{Clinical Report}

\section{DENTIGEROUS CYST: A RARE PRESENTATION}

\section{Chandra Shekhar,* Kranti Bhavana,** Sarita Kumari Mishra**}

Abstract: Though Dentigerous cysts are encountered not so frequenty by the otolaryngologist, a giant dentigerous cyst causing facial deformity and requiring a major reconstruetive surgery is very rare. One such case is described.

Keywords: Dentigerous cyst, Facial asymmetry, Reconstructive surgery.

\section{INTRODUCTION}

The dentigerous cyst, also known as the follicular cyst is defined as a cyst which envelops the whole or part of the crown of an unerupted tooth and is attached to its neck. Majority of these cysts are discovered accidentally on routine radiographs, otherwise they escape detection and enlarge to produce expansion of the jaw. ${ }^{(1)}$ The present case is being reported because of its rare mode of presentation.

*Associate Professor and Head of the Dept., Dept. of ENT, **Post Graduate Student- Final year, Patna Medical College, Patna.

Indian Journal of Otolaryngology and Head and Neck Surgery Vol. 58, No. 4, October - December 2006- 